Published in final edited form as:

BMJ Support Palliat Care. 2016 June ; 6(2): 160-169. doi:10.1136/bmjspcare-2014-000819.

\title{
A systematic review and meta-analysis of meditative interventions for informal caregivers and health professionals
}

\author{
Marisa Dharmawardene ${ }^{1}$, Jane Givens ${ }^{2,3}$, Amy Wachholtz $^{4}$, Suzana Makowski ${ }^{5}$, and \\ Jennifer Tjia ${ }^{5,6}$ \\ ${ }^{1}$ Division of Palliative Medicine, University of Calgary, Alberta, Canada \\ ${ }^{2}$ Division of Gerontology, Harvard Medical School, Boston, Massachusetts, USA \\ ${ }^{3}$ Hebrew SeniorLife Institute for Aging Research, Boston, Massachusetts, USA \\ ${ }^{4}$ Department of Psychiatry, University of Massachusetts Medical School, Worcester, \\ Massachusetts, USA \\ ${ }^{5}$ Division of Palliative Medicine, University of Massachusetts Medical School, Worcester, \\ Massachusetts, USA \\ ${ }^{6}$ Division of Epidemiology and Chronic Diseases, University of Massachusetts Medical School, \\ Worcester, Massachusetts, USA
}

\section{Abstract}

Background-Burnout, stress and anxiety have been identified as areas of concern for informal caregivers and health professionals, particularly in the palliative setting. Meditative interventions are gaining acceptance as tools to improve well-being in a variety of clinical contexts, however, their effectiveness as an intervention for caregivers remains unknown.
Aim-To explore the effect of meditative interventions on physical and emotional markers of well-being as well as job satisfaction and burnout among informal caregivers and health professionals.

Design-Systematic review of randomised clinical trials and pre-post intervention studies with meditative interventions for caregivers.

\footnotetext{
Correspondence to: Dr Jennifer Tjia, Division of Epidemiology and Chronic Diseases, University of Massachusetts Medical School, Albert Sherman Building, 368 Plantation Street, AS8-2068, Worcester, MA 01605, USA; ; Email: jennifer.tjia@ umassmed.edu.

Additional material is published online only. To view please visit the journal online (http://dx.doi.org/10.1136/ bmjspcare-2014-000819).

National Presentations: Parts of this study have been accepted for presentation at the International Symposium for Contemplative Studies in Boston, MA, 30 October to November 2014.

Contributors JT and SM were involved in the project conception. JT, SM and AW obtained funding. JT, JG and MD obtained data, conducted analysis, and drafted the manuscript. MD, JT, JG and AW were involved in the interpretation of data analysis. All authors were involved in the critical revision of manuscript.

Competing interests None.

Provenance and peer review Not commissioned; externally peer reviewed.

Data sharing statement Detailed summary information from full text review of individual articles included in systematic review/ meta-analysis and overall forest plots for all measured outcomes have been included as supplemental files for possible web publication rather than the article itself. Search strings for all databases used for the review are available as additional unpublished data.
} 
Data sources-PubMed, EMBASE, CINAHL and PsycINFO were searched up to November 2013. Of 1561 abstracts returned, 68 studies were examined in full text with 27 eligible for systematic review.

Results-Controlled trials of informal caregivers showed statistically significant improvement in depression (effect size 0.49 (95\% CI 0.24 to 0.75 )), anxiety (effect size 0.53 (95\% CI 0.06 to 0.99 )), stress (effect size 0.49 (95\% CI 0.21 to 0.77 )) and self-efficacy (effect size 0.86 (95\% CI 0.5 to 1.23$)$ ), at an average of 8 weeks following intervention initiation. Controlled trials of health professionals showed improved emotional exhaustion (effect size 0.37 (95\% CI 0.04 to 0.70)), personal accomplishment (effect size 1.18 (95\% CI 0.10 to 2.25)) and life satisfaction (effect size 0.48 (95\% CI 0.15 to 0.81$)$ ) at an average of 8 weeks following intervention initiation.

Conclusions-Meditation provides a small to moderate benefit for informal caregivers and health professionals for stress reduction, but more research is required to establish effects on burnout and caregiver burden.

\section{BACKGROUND}

Meditative interventions are increasingly accepted as a method of reducing stress and improving well-being among patients with chronic illness. ${ }^{1-3}$ Such interventions can encompass a range of techniques including open awareness meditation, focused meditation, mindfulness meditation, mantra-based meditation and integrative body movement traditions such as yoga or tai chi. In clinical medicine, mindfulness programmes that incorporate a combination of these techniques are well-known, such as the Mindfulness-Based Stress Reduction (MBSR) programme developed by Jon Kabat-Zinn. ${ }^{4}$ Such programmes teach persons how to cope with stress, pain, and illness by paying moment-to-moment attention to aspects of daily life in an intentional, non-judgmental and non-reactive way. While recent attention has focused on summarising the effects of meditative interventions in clinical areas in general, ${ }^{2}$ the independent effect of meditative interventions on caregivers has not been well described.

Informal caregivers, defined as unpaid persons who provide care for a relative or close friend, are present in one of every five households. ${ }^{5}$ Caregiving is known to exact a toll on informal caregivers' well-being and has been associated with increased levels of depression and anxiety, poorer self-reported physical health, compromised immune function and increased mortality. ${ }^{5}$ Not surprisingly, healthcare professionals providing direct patient care also report distress attributable to their professional responsibilities, including emotional exhaustion, ${ }^{6-8}$ burnout ${ }^{9}$ and substance abuse. ${ }^{10}$ Meditative interventions may hold promise for professional and informal caregiver populations, but their effectiveness has not been summarised. One systematic review from 2009 addresses the effect of mindfulness on health professionals, ${ }^{11}$ but does not include informal caregivers and includes only the MBSR intervention. In addition, we know little about the potential benefit of meditative interventions for caregivers who specifically serve palliative care and hospice patients, populations highly dependent on informal caregivers and highly stressful to healthcare professionals. A recent review of evidence for interventions to improve palliative care for seriously-ill patients did not address either caregivers issues or mindfulness. ${ }^{12}$ 
To address these gaps, we conducted a systematic literature review of meditative interventions for informal caregivers and health professionals. Our intent is to describe the extent to which randomised clinical trials (RCTs) and pre-post studies have evaluated the effect of mindfulness interventions on psychological, emotional and physical measures of distress and well-being in these populations. In addition, we sought to explore the impact of these interventions on less common outcomes for health professionals (burnout, selfefficacy, job satisfaction) and informal caregivers (grief and bereavement, caregiving selfefficacy, and caregiver burden), as well as among caregivers of patients with advanced illness.

\section{METHODS}

\section{Information sources and search}

PubMed, EMBASE, CINAHL and PsycINFO were initially searched up to 24 June 2013, and updated on 17 November 2013. Studies were not excluded based on date of publication. The PubMed search strategy (see appendix) used a combination of medical subject heading $(\mathrm{MeSH})$ terms and text keywords. This approach was also employed for the other databases, keeping subject headings and key words as similar as possible between the search strings. There is no registered review protocol for this study.

\section{Eligibility criteria and study selection}

For the purpose of this review, studies with a meditation intervention (defined as a form of mental training that requires either stilling or emptying the mind, and that has as its goal a state of "detached observation" in which practitioners are aware of their environment, but do not become involved in thinking about it or a state of intentional, deep contemplation of a specific idea, passage, or object) ${ }^{3}$ were eligible. These include mantra meditation (eg, Transcendental Meditation, Relaxation Response) and mindfulness meditation (eg, Vipassana, Zen Buddhist meditation, MBSR and Mindfulness-Based Cognitive Therapy) according to categorisations published in an Agency for Healthcare Research and Quality (AHRQ) review of meditation practices for health. ${ }^{3}$ While AHRQ's categorisation also includes movement-based practices (eg, yoga asana practice, tai chi or qigong), these were excluded from our review because we focused on explicitly cognitively based meditation interventions.

One investigator (MD) reviewed all abstracts from the search results and selected studies for full text review if they were pre-post studies or RCTs focusing on health professional caregivers or informal caregiver populations. Health professional caregivers were defined as clinical healthcare personnel involved in direct patient care (ie, physicians, residents, nurses, nursing aides and nursing staff ). Informal caregivers were defined as untrained caregivers providing direct patient care. We excluded studies that were dissertations, letters to the editor, posters or conference presentations, not published in English or had fewer than five participants. At least two investigators (MD, JG or JT) independently reviewed the full texts of the resulting articles for final inclusion. At this stage we excluded studies that included healthcare employees in general but did not specifically report the effect on healthcare providers with direct patient care responsibilities. We also excluded multicomponent 
interventions that did not separately report the effects of any meditative components.

Differences were resolved by consensus. We reviewed the reference lists of all studies that were reviewed for any other original studies that were missed in the original search.

\section{Data extraction}

We extracted the following information for each study: author; year of publication; country; study design; caregiver population characteristics (mean age, health professional occupation, hours of caregiving for informal caregivers, diagnoses for which the patient is receiving care); sample size; intervention and control (when applicable) characteristics (material covered and hours of instruction); and outcome measures (physiological and nonphysiological). Data were extracted to an electronic spreadsheet by one author (MD) and independently reviewed by two other authors ( JG and JT).

\section{Risk of bias in individual studies and across studies}

Study quality was independently evaluated by two study investigators using criteria by Downs and Black. ${ }^{13}$ Investigators independently assigned scores by evaluating factors that could systematically bias the results including blinding, recruitment, randomisation and analytic approach. Differences in scores were resolved by reviewing the entire Downs and Black checklists of each study to achieve consensus.

\section{Data analysis}

To construct a meta-analysis of the study results, we grouped studies by caregiver group (ie, informal caregivers and healthcare providers), as well as negative and positive outcome domains. Negative outcomes included: mood (depression, anxiety); stress; caregiver burden; fatigue; energy; sleep; confusion; and grief. Positive outcomes included: self-efficacy; hope; life satisfaction; and quality of life (mental and physical). Healthcare provider studies also reported outcomes for job satisfaction, provider burnout (including subscales for emotional exhaustion, depersonalisation and personal accomplishment) ${ }^{14}$ and patient safety events. Because of the clinical heterogeneity of the physiological measures, we did not conduct meta-analyses of these outcomes, but describe the findings of these studies in the results.

When there were at least three studies within an outcome domain, we calculated the standardised difference in mean change scores as a continuous variable. For controlled trials, this was a difference in difference measurement of the standardised change in mean scores before and after the intervention among the experimental group relative to the control group. For pre-post studies without a comparator group, this difference measurement was the standardised change in scores post-intervention relative to preintervention only. We were unable to report the relative change score for selected domains in several studies owing to incompletely reported data. These included for depression and SF36 scores in Black et al, ${ }^{15}$ stress in Severtsen and Bruya ${ }^{16}$ and Danucalov et al, ${ }^{17}$ and the individual components of the Maslach Burnout Index in the Shapiro study. ${ }^{18}$

We assessed publication bias by creating funnel plots ${ }^{19}$ within each outcome group by caregiver type; we report only funnel plots when there was evidence of publication bias. We used the Q statistic to assess heterogeneity of effect estimates within each outcome group by 
caregiver type, and random-effects models to estimate standardised mean differences for each outcome with an associated $95 \%$ CI. ${ }^{20}$ We graphically display the combined effect size estimates from the random-effects models for each outcome domain for informal caregivers and healthcare providers separately, grouping controlled trials and pre-post intervention studies separately within each group. We used Comprehensive Meta-Analysis (Biostat, Inc, Englewood, NJ) to conduct these analyses.

\section{RESULTS}

\section{Study selection}

Our searches of PubMed, EMBASE, CINAHL and PsycINFO produced 2912 articles (2051 articles from initial mindfulness searches and 861 articles classified as 'relaxation response'). Following removal of duplicates and screening of articles based on titles and abstracts, 65 studies remained. An additional three papers were found in citation review, resulting in a total of 68 papers examined in full text. Twenty-seven of these met our criteria for inclusion in the review and meta-analysis (figure 1).

\section{Characteristics of meditative and comparator group interventions}

A summary of major study characteristics is provided in table 1. For health professionals and informal caregiver studies, interventions ranged in duration from 4 to 8 weeks. Eight studies used a traditional MBSR intervention ( 8 weeks including a mix of mindfulness meditation, Hatha yoga, walking meditation and one full day retreat). ${ }^{1821-27}$ Five studies used a modified MBSR programme with shortened class times (eg, 60-90 min classes), study duration, or omission of the full-day retreat. ${ }^{28-32}$ Six studies used mindfulness-based programmes that were not MBSR, but included a similar mix of instruction in mindfulness meditation, yogic movement and other interventions (ie, appreciative inquiry). ${ }^{1733-37}$ Five studies used yogic meditation and chanting practices ${ }^{151638-40}$ and one study (reported in three separate papers) used passage meditation involving contemplation on a sacred text. ${ }^{41-43}$ One study used a biofeedback-assisted meditation programme ${ }^{44}$ and another used a practice derived from transcendental meditation called Benson relaxation training. ${ }^{45}$ Of the 12 controlled trials, 6 used a waitlist control. ${ }^{18222841-43}$ Other control group interventions included stress management strategies, ${ }^{29}$ music relaxation ${ }^{1538-39}$ and self-care training. ${ }^{2734}$

\section{Characteristics of patient populations served}

The majority of the informal caregiver studies (11/15) focused on caregivers of patients with dementia. Other studies focused on caregivers of patients with advanced cancer, ${ }^{32}$ children with chronic illness, ${ }^{25}$ children utilising special education programmes ${ }^{37}$ and other adult patient populations. ${ }^{26}$ The amount of care provided in order to be identified as a caregiver varied considerably between studies, ranging from $>2 \mathrm{~h}$ per day ${ }^{44}$ to live-in caregivers. ${ }^{40}$ Four studies required that caregivers be identified as the primary caregiver for the patient in order to be included in the study. ${ }^{152738-39}$ Patients receiving care in the 12 health professional studies included behavioural health unit inpatients, critically ill hospitalised patients, geriatric long-term care patients as well as patients drawn from anywhere within a given health or hospital system. 
Few studies specifically evaluated caregivers of patients with advanced illness. Only 3 of the 12 professional health caregiver studies utilised caregiving settings with potential relevance to palliative care. ${ }^{283044}$ Of 15 informal caregiver studies, 12 targeted patient populations potentially relevant to palliative medicine populations with advanced disease, including 11 studies in dementia populations and 1 study in a cancer, but none specifically focused on palliative medicine or hospice settings.

The following presents results by study populations of informal caregivers and health professionals separately. Results for individual studies are shown in the online supplementary appendix, eTables 1 and 2.

\section{Studies of informal caregivers}

Study characteristics-Of the 15 informal caregiver studies, 7 were RCTs, 1 was a nonrandomised controlled trial and 7 were pre-post intervention studies. Sample sizes ranged from 9 to 78. Overwhelmingly, informal caregivers were female, with only 1 study having a majority of male caregivers. ${ }^{39}$ One study used waitlist controls,,${ }^{37} 1$ had an inactive control group ${ }^{17}$ and 6 had active controls. ${ }^{1526273438-39}$ The median Downs and Black study quality score was 11 (range 7-17).

Interventions effects, by outcome measurements-Self-reported measures of stress and anxiety were used in eight of the informal caregiver studies, with seven noting statistically significant improvement with meditative intervention. ${ }^{17252732343740}$ Mood or mental health scales were used in most (12 of 15) informal caregiver studies, with the majority ( 8 of 12) reporting statistically significant improvement after a mindfulness intervention. ${ }^{1725273234363740}$ Heterogeneity Q statistics were statistically significant for controlled trials of anxiety, and for pre-post intervention studies for depression (tables 2 and 3 ). The combined effect for controlled trials of depression, anxiety, stress, self-efficacy and quality of life/mental health suggest benefit from meditation (table 2), as does the combined effect for pre-post interventions for depression, anxiety, stress and caregiver burden (table 3). There were an insufficient number of studies to examine the combined effect for some negative caregiver outcomes, including: fatigue; ${ }^{3440}$ sleep quality; 3440 confusion; ${ }^{2540}$ and grief. ${ }^{23}$ There were also an insufficient number of studies to examine the combined effect of some positive outcomes such as personal growth and hope, ${ }^{3137}$ energy ${ }^{3238}$ and life satisfaction. ${ }^{26}$

Among studies with positive outcome domains, six studies measured self-efficacy with three finding a significant improvement in self-efficacy. ${ }^{263145}$ The combined effect of controlled trials for self-efficacy suggests benefit from meditation, but not for reduction in caregiver burden in either controlled trials (table 2).

Seven (7) informal caregiver studies employed physiological measures of stress including blood pressure, ${ }^{40}$ cortisol, ${ }^{173234}$ interleukin- $6,{ }^{3234}$ tumour necrosis factor, ${ }^{34} \mathrm{C}$ reactive protein, ${ }^{34} \mathrm{NF}-\kappa \mathrm{B}$ transcription factor, ${ }^{15}$ interferon regulatory factor- $1,{ }^{15}$ telomerase activity ${ }^{38}$ and regional cerebral metabolism..$^{38}$ Of these seven, six demonstrated a statistically significant change in physiological testing postintervention. ${ }^{15173238-40}$ 


\section{Studies of health professionals}

Study characteristics-Of 12 health professional studies, 6 were controlled trials and 6 were pre-post trials. Sample sizes varied from 8 to 93 . Four studies used waitlist controls, five had no controls and three had active controls that included leadership exercises, ${ }^{29}$ stress relaxation exercises ${ }^{30}$ and aerobic exercise. ${ }^{16}$ The median Downs and Black study quality summary score was 12 (range 6-18).

Health professional studies most commonly included nurses ${ }^{161821222428-303541-44}$ with nurses studied exclusively in five papers. ${ }^{1528-3044}$ Only one study ${ }^{33}$ focused exclusively on physicians and one other separated physician participants into a separate group for analysis. ${ }^{24}$ The remaining six health professional studies were open to a variety of different caregivers with resultant mixed samples that included physicians and nurses as well as psychologists, physiotherapists and social workers.

Interventions effects, by outcome measurements-Of the five health professional studies reporting non-physiological measures of stress, all found statistically significant improvement in stress levels postmindfulness intervention. ${ }^{1821354144} \mathrm{One}^{44}$ of two ${ }^{2944}$ studies for anxiety reported significant improvement as well. Health professional caregivers reported increased conscientiousness, ${ }^{33}$ increased empathy towards patients, ${ }^{3343}$ and increased compassion towards themselves. ${ }^{18} \mathrm{~A}$ statistically significant increase in the perception of job-related personal accomplishment was also noted in one ${ }^{28}$ of two studies. ${ }^{2841}$ While one study reported reductions in patient safety events such as aggression, falls, and medication errors, these were not statistically analysed. ${ }^{21}$ There were insufficient number of studies to examine the combined effect of meditation on healthcare professional empathy, ${ }^{3343}$ job satisfaction ${ }^{2841}$ or patient safety events. ${ }^{21}$

Both studies utilising mental health scales found statistically significant improvements in self-reported mental health after mindfulness training. ${ }^{2441}$ Heterogeneity Q statistics were statistically significant for controlled trials of self-efficacy (tables 4 and 5). The combined effect of controlled trials for emotional exhaustion and personal accomplishment suggests benefit from meditation (table 4), as do pre-post interventions targeting emotional exhaustion, depersonalisation, personal accomplishment and stress (table 5).

Two studies ${ }^{1841}$ reporting outcomes of self-efficacy, and the study of self-compassion ${ }^{43}$ reported statistically significant improvements post-mindfulness intervention. Four studies reported effects of meditation on life satisfaction, ${ }^{18283042}$ with all four studies showed promising improvements with meditation, with a statistically significant combined effect in the random effects model (table 4). The heterogeneity Q statistic for controlled trials of selfefficacy was statistically significant, with the combined effect for self-efficacy suggesting benefit from meditation among controlled trials (table 4).

One health professional study used a physiological measure of stress. ${ }^{16}$ This study used EEG measurement of the proportion of $\alpha$ and $\beta$ waves produced with stress-reduction interventions, and did not find a statistically significant difference between intervention groups. All outcome domains for both caregiver groups are listed in the table of Overall Forrest Plots (see online supplementary file). 


\section{DISCUSSION}

Our systematic review revealed 27 studies evaluating the effect of meditative intervention studies on caregivers, including 12 for health professionals and 15 for informal caregivers. All studies evaluated the effect of such interventions on psychological, emotional, or physical well-being, but specific outcome measures differed between each caregiver group. The only outcome with statistically significant improvements for informal caregivers and health professionals in combined analyses was self-efficacy. Otherwise, the specific outcome measures with sufficient data for combined synthesis differed among each care provider group.

For informal caregivers, there was evidence from controlled trials of statistically significant improvements in mood (depression and anxiety) and stress. Pre-post studies, but not controlled trials, showed improvements in caregiver burden, and there were insufficient studies to comment on outcomes of energy, fatigue, hope, confusion, sleep, grief or life satisfaction. We also did not find any studies examining the effect of meditation on outcomes such as caregiver capacity for decision-making, patient advocacy or resilience. There was no available information in any of the studies on patient care or well-being that may be mediated by improvements in caregiver outcomes.

Among health professional caregivers, meditative interventions demonstrated statistically significant improvement in participant's level of emotional exhaustion, personal accomplishment, self-efficacy and life satisfaction in controlled trials. Feelings of depersonalisation were improved in pre-post studies but not in controlled trials. There were an insufficient number of controlled trials to comment on whether meditation improves stress, but pre-post studies suggested significant stress reduction. Insufficient studies were available to comment on job satisfaction, mental health, physical quality of life, depression, anxiety, empathy or resilience. There were also an inadequate number of studies to summarise results on job performance or patient outcomes.

Our review revealed several notable gaps in the literature that are worthy of comment. In terms of populations studied, the vast majority of studies enrolled female participants, which makes generalisation to male caregivers difficult. We noted a paucity of studies specifically evaluating caregivers of patients with advanced illness, those at the end-of-life, or patients on hospice. Methodologically, we found that many studies relied on self-report of adherence; studies also displayed high variability in terms of study quality as determined by the Downs and Black score. Many outcome domains have not been studied at all or very little, including patient outcomes (eg, safety events, patient satisfaction), patient advocacy by family caregivers, and family grief. Finally, we also noted considerable variability in the reporting of the duration of study effect.

The findings of our study should be interpreted in the context of several limitations. Our search was limited to articles published in English. Owing to variations in how meditative interventions are reported, we recognise the possibility that we have omitted some studies that did not match our search terms but which may have been appropriate for this review. However, we anticipate that this possibility is small, due to our strategy of searching the 
citations of all articles found. The total number of studies eligible for review was small, raising the possibility of selection bias and potentially limiting the strength of our results and recommendations. Some outcome domains did not have enough studies for the metaanalysis. While some would argue that it is possible to combine results from as few as two studies ${ }^{46}$ others have shown that meta-analyses with very small numbers of studies tend to underestimate heterogeneity. ${ }^{47}$ Despite our minimum requirement of three studies per outcome domain, our analysis groups remain objectively small, thus interpretation of heterogeneity results should still be approached with caution. We used Cochran's Q statistic to measure heterogeneity, which is limited by low power when analysing a small number of studies. While the $\mathrm{I}^{2}$ index does provide an alternative measure of the extent of heterogeneity in meta-analyses, it is also limited by lower power with a small number of studies. $^{48}$

\section{Next steps/conclusions}

Our review suggests several considerations for future research utilising meditative interventions among caregivers. Since the majority of interventions utilise cognitive and movement based aspects, and because there is substantial variation within each of these categories (eg, mindfulness meditation, focused concentration, open awareness, body/ internal focus, nature/external focus, yoga, tai chi, qigong), it may be useful for future studies to identify the beneficial outcomes associated with specific techniques, or the most appropriate target audiences for each technique. Future studies should be limited to those with rigorous study designs, including appropriate control interventions and adequate power. Further small, underpowered trials will detract from the ability to produce unambiguous results in future analyses. Lastly, it would be helpful for future studies to address the question of how long an intervention needs to be in order to have a measureable effect, and how long those effects are likely to last. This question of effective intervention duration is particularly relevant to caregiver populations of patients in hospice or at the end of life, as informal caregivers of these patients are often unable to participate in programmes of high intensity or duration.

\section{Supplementary Material}

Refer to Web version on PubMed Central for supplementary material.

\section{Acknowledgements}

The authors would like to thank Carol Mita, Reference and Research Services Librarian of Countway Library of Medicine, who helped to design the database search strategy, and Bei Chang, PhD, Biostatistics, Quantitative Health Sciences at the University of Massachusetts Medical School who reviewed an earlier version of this manuscript. Neither individual was compensated for their time.

Funding This work was supported by a Gold Foundation Prize for Humanism in Medicine. JG was supported by NIH-NIA K23 AG034967. AW was supported by NIH-NIDA K23 DA030397.

\section{REFERENCES}

1. Grossman P, Niemann L, Schmidt S, et al. Mindfulness-based stress reduction and health benefits. A meta-analysis. J Psychosom Res. 2004; 57:35-43. [PubMed: 15256293] 
2. Goyal M, Singh S, Sibinga ES, et al. Meditation programs for psychological stress and well-being: a systematic review and meta-analysis. JAMA Intern Med. 2014; 174:357-368. [PubMed: 24395196]

3. Ospina, MB.; Bond, TK.; Karkhaneh, M., et al. (Prepared by the University of Alberta Evidencebased Practice Center under Contract No. 290-02-0023.) AHRQ Publication No. 07-E010. Rockville, MD: Agency for Healthcare Research and Quality; 2007 Jun. Meditation Practices for Health: State of the Research. Evidence Report/Technology Assessment No. 155.

4. Kabat-Zinn J. An outpatient program in behavioral medicine for chronic pain patients based on the practice of mindfulness meditation: theoretical considerations and preliminary results. Gen Hosp Psychiatry. 1982; 4:33-47. [PubMed: 7042457]

5. Centers for Disease Control and Prevention and the Kimberly-Clark Corporation. Assuring Healthy Caregivers, A Public Health Approach to Translating Research into Practice: The RE-AIM Framework. Neenah, WI: Kimberly-Clark Corporation; 2008. http://www.cdc.gov/aging/ (last accessed 27 Jun 2014)

6. Shanafelt TD, Bradley KA, Wipf JE, et al. Burnout and self-reported patient care in an internal medicine residency program. Ann Intern Med. 2002; 136:358-367. [PubMed: 11874308]

7. Shanafelt TD, Sloan JA, Habermann TM. The wellbeing of physicians. Am J Med. 2003; 114:513519. [PubMed: 12727590]

8. Shanafelt TD, Novotny P, Johnson ME, et al. The well-being and personal wellness promotion strategies of medical oncologists in the North Central Cancer Treatment Group. Oncology. 2005; 68:23-32. [PubMed: 15775690]

9. Spickard A Jr, Gabbe SG, Christensen JF. Midcareer burnout in generalist and specialist physicians. JAMA. 2002; 288:1447-1450. [PubMed: 12243624]

10. O'Connor PG, Spickard A Jr. Physician impairment by substance abuse. Med Clin North Am. 1997; 81:1037-1052. [PubMed: 9222267]

11. Irving JA, Dobkin PL, Park J. Cultivating mindfulness in health care professionals: a review of empirical studies of mindfulness-based stress reduction (MBSR). Complement Ther Clin Pract. 2009; 15:61-66. [PubMed: 19341981]

12. Dy, SM.; Aslakson, R.; Wilson, RF., et al. Evidence Report No. 208. (Prepared by the Johns Hopkins University Evidence-based Practice Center under Contract No. 290-2007-10061-I.) AHRQ Publication No. 12(13)-E014-EF. Rockville, MD: Agency for Healthcare Research and Quality; 2012 Oct. Improving Health Care and Palliative Care for Advanced and Serious Illness. Closing the Quality Gap: Revisiting the State of the Science. http:// www.effectivehealthcare.ahrq.gov/reports/final.cfm

13. Downs SH, Black N. The feasibility of creating a checklist for the assessment of the methodological quality both of randomised and non-randomised studies of health care interventions. J Epidemiol Community Health. 1998; 52:377-384. [PubMed: 9764259]

14. Maslach, C.; Jackson, SE.; Leiter, MP. Maslach burnout inventory. 3rd edn.. Palo Alto, CA: Consulting Psychologists Press; 1996.

15. Black DS, Cole SW, Irwin MR, et al. Yogic meditation reverses NF- $\kappa$ B and IRF-related transcriptome dynamics in leukocytes of family dementia caregivers in a randomized controlled trial. Psychoneuroendocrinology. 2013; 38:348-355. [PubMed: 22795617]

16. Severtsen B, Bruya MA. Effects of meditation and aerobic exercise on EEG patterns. J Neurosci Nurs. 1986; 18:206-210. [PubMed: 2943837]

17. Danucalov MA, Kozasa EH, Ribas KT, et al. A yoga and compassion meditation program reduces stress in familial caregivers of Alzheimer's disease patients. Evid Based Complement Alternat Med. 2013; 2013:513149. [PubMed: 23690846]

18. Shapiro SL, Astin JA, Bishop SR, et al. Mindfulness-based stress reduction for health care professionals: results from a randomized trial. Int J Stress Management. 2005; 12:164-176.

19. Egger M, Smith GD, Schneider M, et al. Bias in meta-analysis detected by a simple, graphical test. BMJ. 1997; 315:629-634. [PubMed: 9310563]

20. DerSimonian R, Laird N. Meta-analysis in clinical trials. Control Clin Trials. 1986; 7:177-188. [PubMed: 3802833] 
21. Brady S, O'Connor N, Burgermeister D, et al. The impact of mindfulness meditation in promoting a culture of safety on an acute psychiatric unit. Perspect Psychiatr Care. 2012; 48:129-137. [PubMed: 22724398]

22. Cohen-Katz J, Wiley SD, Capuano T, et al. The effects of mindfulness-based stress reduction on nurse stress and burnout, Part II: a quantitative and qualitative study. Holist Nurs Pract. 2005; 19:26-35. [PubMed: 15736727]

23. Epstein-Lubow G, McBee L, Darling E, et al. A pilot investigation of mindfulness-based stress reduction for caregivers of frail elderly. Mindfulness. 2011; 2:95-102.

24. Goodman MJ, Schorling JB. A mindfulness course decreases burnout and improves well-being among healthcare providers. Int J Psychiatry Med. 2012; 43:119-128. [PubMed: 22849035]

25. Minor HG, Carlson LE, Mackenzie MJ, et al. Evaluation of a Mindfulness-Based Stress Reduction (MBSR) program for caregivers of children with chronic conditions. Soc Work Health Care. 2006; 43:91-109. [PubMed: 16723337]

26. Van Puymbroeck M. The influence of mindfulness-based stress reduction and walking on the psychological well-being of female informal caregivers: a pilot study. Am J Recreation Therapy. 2010; 9:15-25.

27. Whitebird RR, Kreitzer MJ, Crain AL, et al. Mindfulness-based stress reduction for family caregivers: a randomized controlled trial. Gerontologist. 2013; 53:676-668. [PubMed: 23070934]

28. Mackenzie CS, Poulin PA, Seidman-Carlson R. A brief mindfulness-based stress reduction intervention for nurses and nurse aides. Appl Nurs Res. 2006; 19:105-109. [PubMed: 16728295]

29. Pipe TB, Bortz JJ, Dueck A, et al. Nurse leader mindfulness meditation program for stress management: a randomized controlled trial. J Nurs Adm. 2009; 39:130-137. [PubMed: 19590469]

30. Poulin PA, Mackenzie CS, Soloway G, et al. Mindfulness training as an evidenced-based approach to reducing stress and promoting well-being among human services professionals. Int J Health Promot Educ. 2008; 46:35-43.

31. Hoppes S, Bryce H, Hellman C, et al. The Effects of Brief Mindfuness Training on Caregivers' Well-Being. Act Adapt Aging. 2012; 36:147-166.

32. Lengacher CA, Kip KE, Barta M, et al. A pilot study evaluating the effect of mindfulness-based stress reduction on psychological status, physical status, salivary cortisol, and interleukin- 6 among advanced-stage cancer patients and their caregivers. J Holist Nurs. 2012; 30:170-185. [PubMed: 22442202]

33. Krasner MS, Epstein RM, Beckman H, et al. Association of an educational program in mindful communication with burnout, empathy, and attitudes among primary care physicians. JAMA. 2009; 302:1284-1293. [PubMed: 19773563]

34. Oken BS, Fonareva I, Haas M, et al. Pilot controlled trial of mindfulness meditation and education for dementia caregivers. J Altern Complement Med. 2010; 16:1031-1038. [PubMed: 20929380]

35. Schenström A, Rönnberg S, Bodlund O. Mindfulness-based cognitive attitude training for primary care staff: a pilot study. J Evid Based Complementary Altern Med. 2006; 11:144-152.

36. Waelde LC, Thompson L, Gallagher-Thompson D. A pilot study of a yoga and meditation intervention for dementia caregiver stress. J Clin Psychol. 2004; 60:677-687. [PubMed: 15141399]

37. Benn R, Akiva T, Arel S, et al. Mindfulness training effects for parents and educators of children with special needs. Dev Psychol. 2012; 48:1476-1487. [PubMed: 22409766]

38. Lavretsky H, Epel ES, Siddarth P, et al. A pilot study of yogic meditation for family dementia caregivers with depressive symptoms: effects on mental health, cognition, and telomerase activity. Int J Geriatr Psychiatry. 2013; 28:57-65. [PubMed: 22407663]

39. Pomykala KL, Silverman DH, Geist CL, et al. A pilot study of the effects of meditation on regional brain metabolism in distressed dementia caregivers. Aging Health. 2012; 8:509-516. [PubMed: 23378856]

40. Innes KE, Selfe TK, Brown CJ, et al. The effects of meditation on perceived stress and related indices of psychological status and sympathetic activation in persons with Alzheimer's disease and their caregivers: a pilot study. Evid Based Complement Alternat Med. 2012; 2012:927509. [PubMed: 22454689] 
41. Oman D, Hedberg J, Thoresen CE. Passage meditation reduces perceived stress in health professionals: a randomized, controlled trial. J Consult Clin Psychol. 2006; 74:714-719. [PubMed: 16881779]

42. Oman D, Richards TA, Hedberg J, et al. Passage meditation improves caregiving self-efficacy among health professionals: a randomized trial and qualitative assessment. J Health Psych. 2008; 13:1119-1135.

43. Oman D, Thoresen CE, Hedberg J. Does passage meditation foster compassionate love among health professionals: a randomised trial. Ment Health Relig Cult. 2010; 13:129-154.

44. Cutshall SM, Wentworth LJ, Wahner-Roedler DL, et al. Evaluation of a biofeedback-assisted meditation program as a stress management tool for hospital nurses: a pilot study. Explore (NY). 2011; 7:110-112. [PubMed: 21397872]

45. Fisher PA, Laschinger HS. A relaxation training program to increase self-efficacy for anxiety control in Alzheimer family caregivers. Holist Nurs Pract. 2001; 15:47-58. [PubMed: 12119919]

46. Valentine JC, Pigott TD, Rothstein HR. How many studies do you need? A primer on statistical power for meta-analysis. J Educ Behav Stat. 2010; 35:215-247.

47. Kontopantelis E, Springate DA, Reeves D. A re-analysis of the Cochrane Library data: the dangers of unobserved heterogeneity in meta-analyses. PLoS ONE. 2013; 8:e69930. [PubMed: 23922860]

48. Huedo-Medina TB, Sanchez-Meca J, Marin-Martinez F, et al. Assessing heterogeneity in metaanalysis: Q statistic or I ${ }^{2}$ index? Psychol Methods. 2006; 11:193-206. [PubMed: 16784338]

\section{APPENDIX}

PubMed Search Strategy:

((“Caregivers"[Majr] OR "Caregivers"[Mesh] OR "Health Personnel"[Mesh:noexp] OR "Nurses"[Mesh] OR "Nursing Staff"[Mesh] OR "Physicians"[Mesh] OR “Nurses”

Aides"[Mesh] OR "Medical Staff"[Mesh] OR caregiver[tiab]) OR caregiving[tiab] OR care giver[tiab] OR care giver/patient[tiab] OR care giver's[tiab] OR care givers[tiab]) OR care giving[tiab] OR nurses[ti] OR physicians[ ti] OR clinicians[ti] OR (health care provider[ti] OR health care provider's[ti] OR health care providers [ti]) OR health care personnel[ti] OR (healthcare provider[ ti] OR healthcare providers[ti]) OR healthcare personnel[ ti] OR hospital staff[ti] OR medical staff[ti] OR nursing staff[ti] OR nursing personnel[ti] AND ("Mind-Body Therapies"[Majr:noexp] OR "Meditation"[Mesh] OR "Yoga"[Mesh] OR "Relaxation Therapy"[Mesh] OR "Mental Healing"[Mesh] OR mindfulness[ tiab] OR contemplative[tiab] OR meditation[tiab] OR yoga[tiab] OR mental healing[tiab] OR mindbody therapies[tiab] OR Relaxation Therapy[tiab]) OR ("relaxation response"[MeSH Major Topic]) OR "relaxation response"[MeSH Terms]) OR "relaxation response"[Title])) OR Benson) AND "relaxation response" 


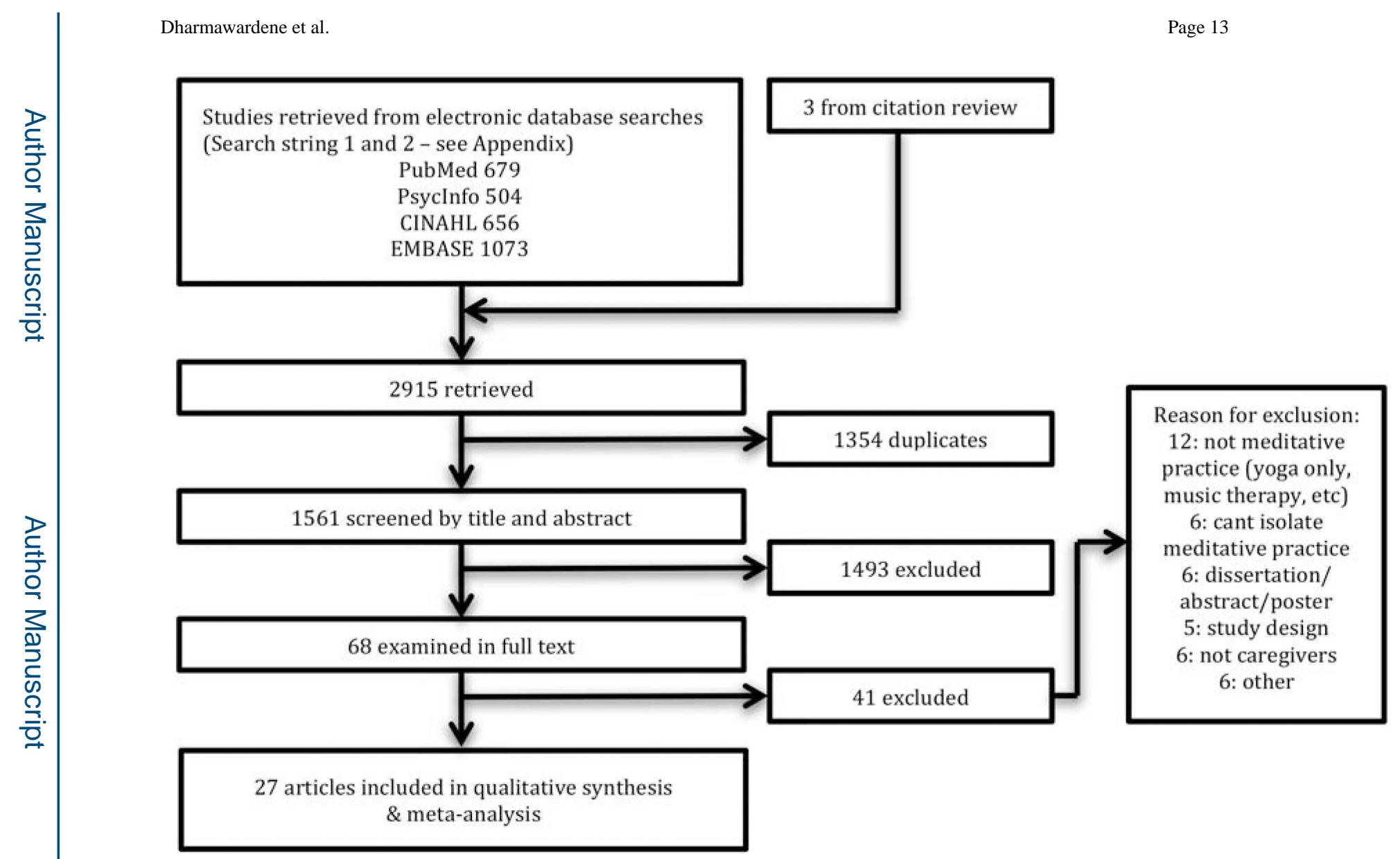

Figure 1.

Article selection process. 
Table 1

Characteristics of reviewed studies, by study population

\begin{tabular}{|c|c|c|}
\hline & $\begin{array}{r}\text { Informal } \\
\text { caregivers } \\
\text { N=15 }\end{array}$ & $\begin{array}{r}\text { Healthcare } \\
\text { professionals } \\
\mathrm{N}=12\end{array}$ \\
\hline \multicolumn{3}{|l|}{ Study site } \\
\hline USA & 12 & 11 \\
\hline Other & 3 & 1 \\
\hline \multicolumn{3}{|l|}{ Study design } \\
\hline Pre-post & 7 & 6 \\
\hline Controlled trial & 8 & 6 \\
\hline \multicolumn{3}{|l|}{ Patient diagnosis } \\
\hline Dementia & 11 & 0 \\
\hline Cancer & 1 & 0 \\
\hline Other & 3 & 12 \\
\hline \multicolumn{3}{|l|}{ Intervention type } \\
\hline Traditional MBSR & 4 & 4 \\
\hline Modified MBSR & 2 & 3 \\
\hline Other mindfulness based programme & 4 & 2 \\
\hline Mantra meditation or chanting & 4 & 1 \\
\hline Other (inclusive Benson relaxation response, meditation $\mathrm{CD}$, passage meditation) & 1 & 2 \\
\hline \multicolumn{3}{|l|}{ Outcome measures } \\
\hline Physiological ${ }^{*}$ & 7 & 1 \\
\hline \multicolumn{3}{|l|}{ Non-Physiological } \\
\hline Stress & 8 & 5 \\
\hline Depression & 13 & 2 \\
\hline Anxiety & 9 & 2 \\
\hline Resilience/caregiver burden & 7 & 0 \\
\hline Compassion/empathy & 2 & 2 \\
\hline Self-efficacy & 6 & 3 \\
\hline Burnout & 0 & 8 \\
\hline
\end{tabular}

Physiological measures include electric encelphalogram, blood pressure, cortisol, telomerase activity, nuclear factor (NF)- $\mathrm{KB}$, interferon response factors (IRF) transcriptome dynamics.

CD, compact disc; MBSR, Mindfulness-Based Stress Reduction. 


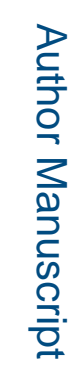

를

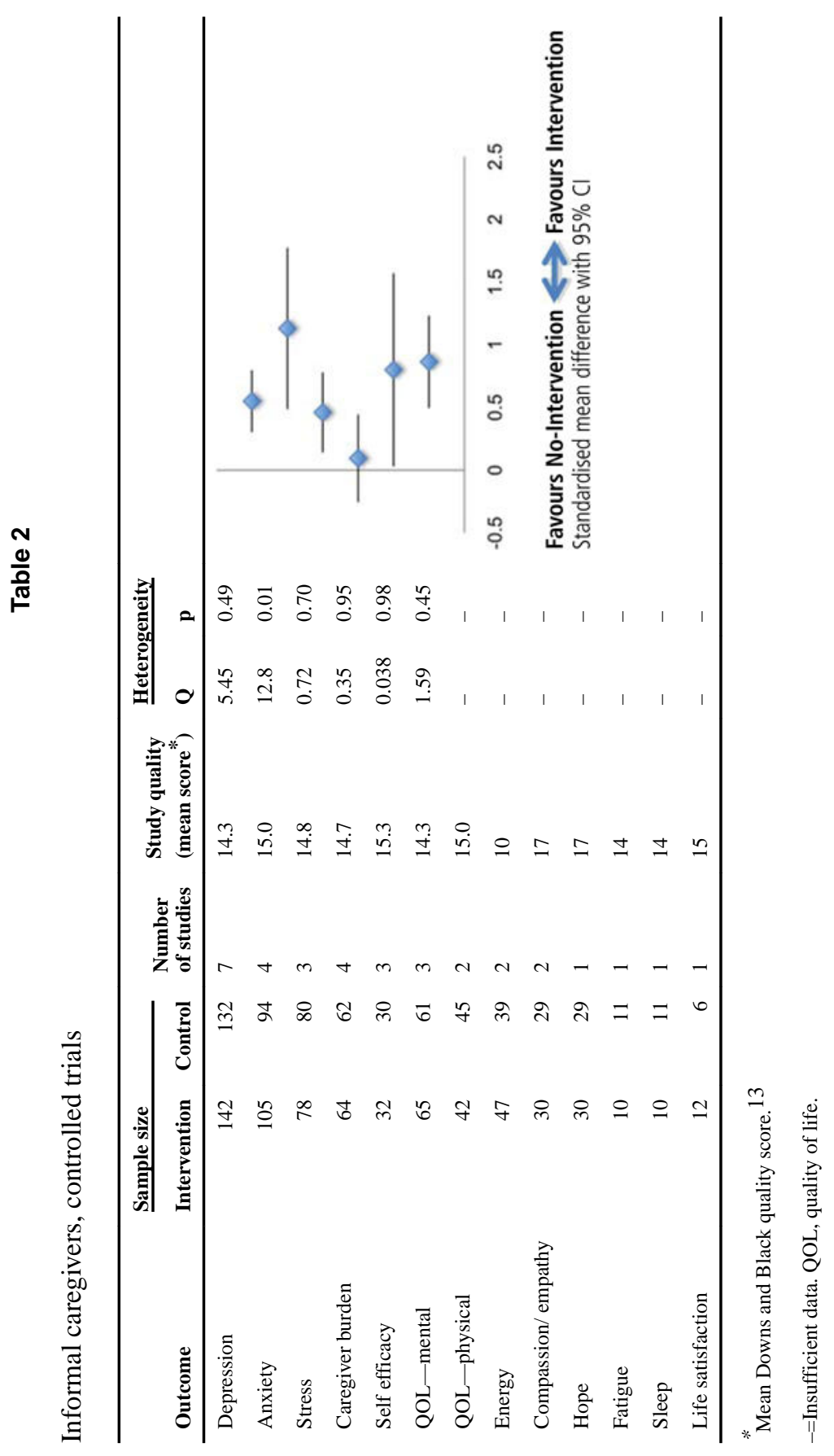

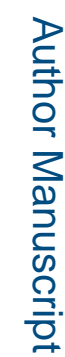

록

일 


\section{로을}

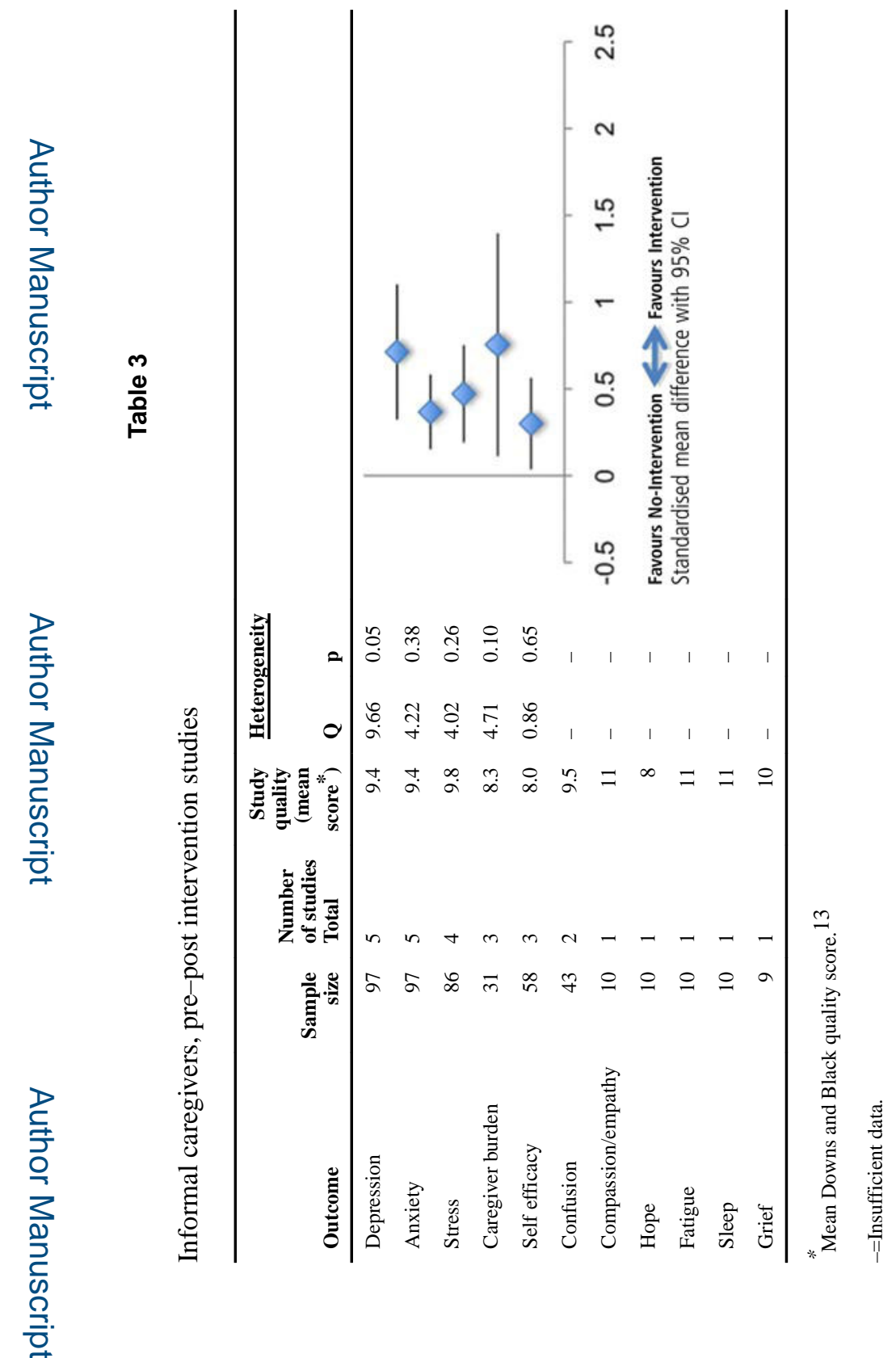

BMJ Support Palliat Care. Author manuscript; available in PMC 2017 June 01. 


\section{로을}

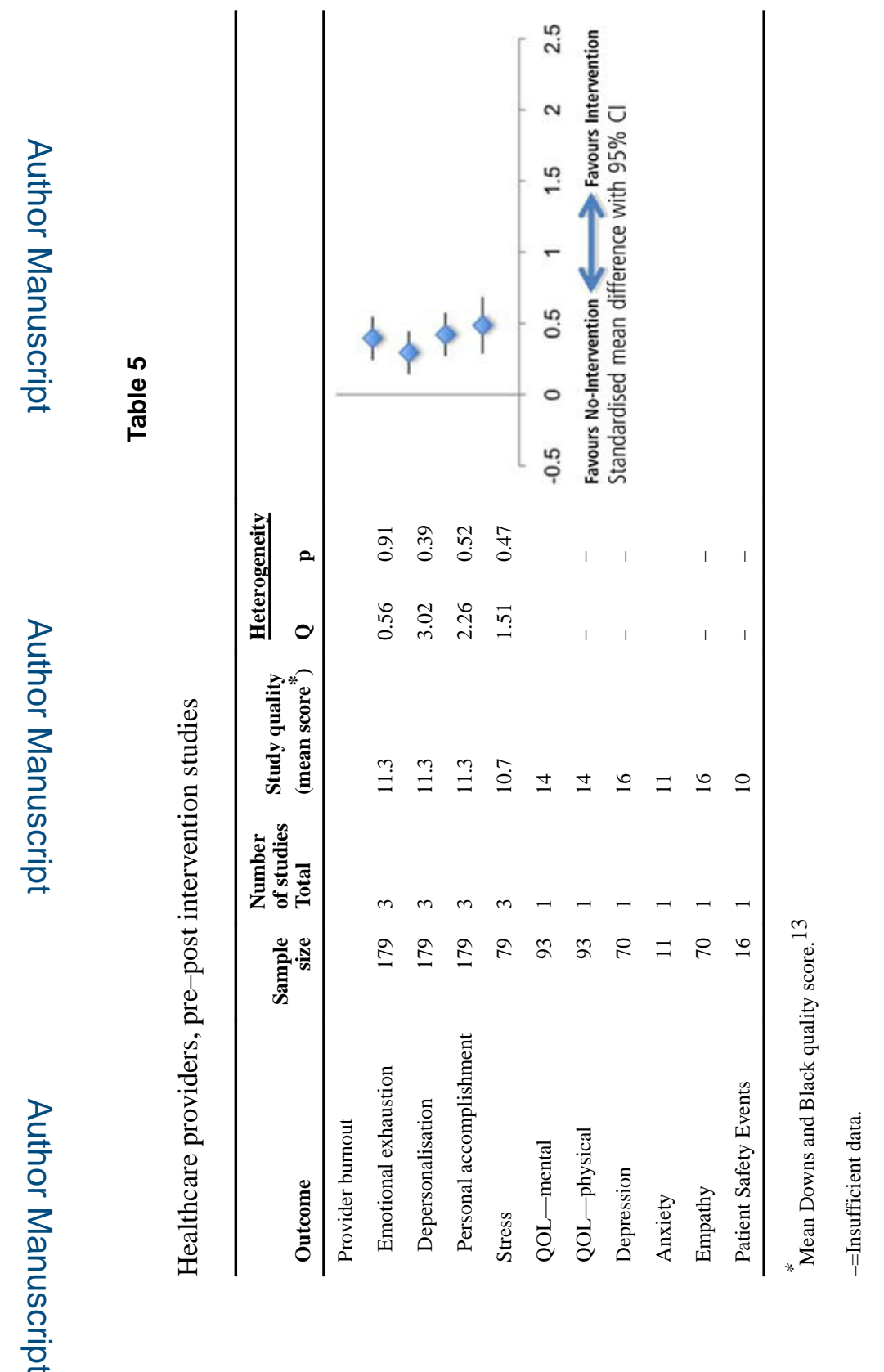

BMJ Support Palliat Care. Author manuscript; available in PMC 2017 June 01. 